УДК 004.832.28

https://doi.org/10.24866/1813-3274/2021-2/42-56

Сейед Махди Сохейли Могадам ${ }^{1}$, Южный федеральный университет,

г. Ростов-на-Дону, Россия

E-mail: soh@sfedu.ru

\title{
СОВЕРШЕНСТВОВАНИЕ АНАЛИТИЧЕСКОГО ИНСТРУМЕНТА РЕSТЕL В СИСТЕМЕ СТРАТЕГИЧЕСКОГО УПРАВЛЕНИЯ ПРОМЫШЛЕННЫМ ПРЕДПРИЯТИЕМ
}

Аннотацияя. Характер решений в области стратегического управления, как правило, сложный и неструктурированный. Соответственно, одной из наиболее важных проблем руководителей стратегического уровня является повышение качества и обоснованности принятия решений. В данном исследовании предпринята попытка решить данные вопросы с помощью нейронных сетей, которые являются одним из наиболее важных методов решения проблем с применением новых вычислительных методов машинного обучения, позволяющих максимизировать выход реакции сложных систем. В рамках исследования осуществляется использование PESTEL в качестве одного из инструментов и фреймворков для анализа внешних факторов, влияющих на промышленные предприятия. Факторы PESTEL обычно выходят за рамки того, на что организация может напрямую влиять. Эти факторы определяют тенденции развития окружающей среды и предоставляют важную информацию для проведения SWOTанализа промышленных предприятий; дают обоснованные гипотезы для разработки стратегии предприятий. Для определения степени и серьёзности воздействия факторов, индикаторов и критериев в работе использовался процесс иерархического анализа и, исходя из количества и серьёзности неопределённостей, в расчётах учитывалось понятие риска. Одним из факторов рассмотренного инструмента стратегического анализа является политический фактор, выступающий одним из самых сложных и неструктурированных в рамках изучаемого метода. Результаты исследования показали, что использование искусственной нейронной сети позволяет провести оценку политического фактора в рамках инструмента

\footnotetext{
${ }^{1}$ Сейед Махди Сохейли Могадам, аспирант 3-го года обучения, кафедра информационной экономики, экономический факультет, Южный федеральный университет, г. Ростов-на-Дону, Россия.

Для ичтирования: Сохейли Могадам Сейед Махди. Совершенствование аналитического инструмента PESTEL в системе стратегического управления промышленным предприятием // АзиатскоТихоокеанский регион: экономика, политика, право. 2021. №. 2. С. 42-56. DOI https://doi.org/10.24866/1813-3274/2021-2/42-56. 
стратегического анализа PESTEL и даёт возможность вырабатывать более обоснованные управленческие решения. Среди двух рассмотренных моделей оптимизатор SGD демонстрирует лучшие результаты по сравнению с оптимизатором ADAM.

Ключевые слова: управление промышленным предприятием, инструменты стратегического управления, PEST-анализ, PESTEL, внешняя среда, анализ политических факторов, система поддержки принятия решений, искусственная нейронная сеть.

Seyed Mahdi Soheili Mogadam ${ }^{1}$, Southern Federal University, Russia

E-mail: soh@sfedu.ru

\section{IMPROVING THE ANALYTICAL TOOL PESTEL IN THE SYSTEM OF STRATEGIC MANAGEMENT OF AN INDUSTRIAL ENTERPRISE}

Abstract. The nature of strategic management decisions is usually complex and unstructured. Accordingly, one of the most important challenges for strategic leaders is improving the quality and soundness of decision-making. In this study, an attempt is made to solve these issues using neural networks, which are one of the most important methods for solving problems using new computational machine learning methods that maximize the response yield of complex systems. The study uses PESTEL as one of the tools and frameworks for analyzing external factors affecting industrial enterprises. PESTEL factors usually go beyond what the organization can directly influence. These factors determine trends in the development of the environment and provide important information for conducting a SWOT analysis of industrial enterprises. To determine the degree and severity of impact factors, indicators and criteria used in the hierarchical analysis process, and, based on the number and severity of uncertainties, the concept of risk was taken into account in the calculations. One of the factors of the considered tool of strategic analysis is the political factor, which is one of the most complex and unstructured within the framework of the studied method. The results of the study showed that the use of a neural network makes it possible to assess the political factor within the framework of the PESTEL strategic analysis tool and makes it possible to develop more informed management decisions. Among the two models reviewed, the SGD optimizer performs better than the ADAM optimizer.

\footnotetext{
1 Seyed Mahdi Soheili Mogadam, 3rd year post-graduate student, Department of Information Economics, Faculty of Economics, Southern Federal University, Rostov-on-Don, Russia.

For citing: Soheili Mogadam Seyed Mahdi. Improving the analytical tool PESTEL in the system of strategic management of an industrial enterprise // PACIFIC RIM: Economics, Politics, Law. 2021. No. 2. P. 42-56. DOI https://doi.org/10.24866/1813-3274/2021-2/42-56.
} 
Keywords: industrial enterprise management, strategic management tools, PEST analysis, PESTEL, external environment, analysis of political factors, decision support system, artificial neural network.

\section{Введение}

Анализ внешней среды в современных условиях должен выступать объективной оценкой меняющегося мира, в котором функционирует промышленное предприятие, с целью создания «системы раннего предупреждения» для выявления потенциальных угроз и стратегических возможностей. Другими словами, актуальность анализа внешней среды хозяйствующего субъекта в промышленности определяется чрезвычайно высокой степенью её динамизма, сложности и неопределенности. Так, главным условием успешного функционирования промышленного предприятия в новой нормальности является способность адаптироваться к изменениям, происходящим во внешней среде, сохраняя при этом устойчивость и жизнеспособность. Организации должны эффективно и своевременно реагировать на новые изменения, происходящие в деловой экосистеме, и выстраивать в соответствии с такими изменениями свои стратегии развития.

Исследователи отмечают, что использование стратегических инструментов в организациях следует рассматривать с точки зрения практики [1]. Это определяется тем, что существует разрыв между теорией относительно того, как следует использовать инструменты и методы стратегического управления, и их реальным применением (со стороны менеджмента промышленных предприятий). Поэтому несколько авторов ввели в научный дискурс новый подход, известный как «стратегия как практика» [2]. В целом, инструменты и методы стратегического управления могут принести много преимуществ организациям при условии, что менеджеры имеют чёткое понимание возможностей и ограничений существующих инструментов и методов [3].

По мнению Ганна и Уильямса, стратегические инструменты могут использоваться для анализа организации и её среды или в качестве механизма улучшения коммуникаций, контроля и координации [1]. Вебстер и др. утверждают, что использование стратегических инструментов и методов управления стратегиями позволяет увеличить аналитические и диагностические навыки менеджеров [4], в то время как Пасанен подчёркивает, что эффективность является наиболее важным преимуществом использования инструментов и методов стратегического управления [5]. Кроме того, согласно Frost: аналитические «методы могут также помочь в представлении сложных проблем и могут рассматриваться как ценные коммуникационные устройства в дополнение к их аналитической роли ... часто становится возможным свести много страниц плана повествования к одной или двум диаграммам, которые являются результатом использования некоторых методов» [6]. 
Отдельно нужно говорить о разном влиянии факторов внешнего окружения на деятельность промышленных предприятий. Так, Ячменева и Вольска [7] в своём исследовании показывают, что политический фактор влияет на инновации промышленных предприятий во всех категориях, таких как рыночная стоимость, устойчивость, надёжность, активность, социальность. Козубикова и др. [8] также доказывают с помощью регрессионного анализа прямое влияние политического фактора на деятельность предприятий в Чехии и Словакии. В обеих странах промышленные предприятии и субъекты малого и среднего предпринимательства больше всего зависят от государственного регулирования и поддержки. Текущее состояние промышленной среды Словакии связано с фундаментальными изменениями в политической и экономической системе, сопровождающими процесс трансформации [8].

Как было отмечено ранее, учёт факторов внешнего окружения становится особенно важным на уровне стратегического управления. Стратегические управленческие решения обычно сложны и неструктурированны [9]. Это определяет важность применения интеллектуальных технологий для обработки больших массивов неструктурированной или слабоструктурированной информации, реализованного в единой среде - системе поддержки принятия решений. Система поддержки принятия решений (СППР) представляет собой совокупность связанных данных и программ, предназначенных для оказания помощи в анализе и принятии решений. Эти системы более эффективны при принятии решений, чем системы управления информацией [10], и сами по себе имеют базу данных, состоящую из существующих знаний о предмете, языка для формулирования вопросов и программ моделирования для проверки возможных решений. Особенности применения СППР в стратегическом управлении промышленным предприятием [11]:

1 - Помощь менеджеру в решении слабо структурированных вопросов;

2 - СППР осуществляется менеджером, эта система не может заменять его;

3 - Повышение эффективности и обоснованности принятия решений.

СППР была разработана для решения проблем информационной системы управления (ИСУ) и также сочетает человеческие ресурсы (знания) с компьютерными возможностями для повышения качества решений, особенно по слабоструктурированным вопросам с использованием данных и моделей. На самом деле, СППР - это двусторонняя компьютерная информационная система, которая является гибкой и адаптивной, используется специально для поддержки решения неструктурированных вопросов управления [12].

Стратегические решения не могут основываться исключительно на субъективных оценках, но должны базироваться на научных исследованиях, статистике и неоспоримой информации. В данном исследовании предпринята попытка использовать систему поддержки для принятия решений стратегическими менеджерами промышленных предприятий при выборе наилучшего решения в рамках инстру- 
ментов управления PESTLE. Содержание принятия стратегических решений реализуется с использованием нейронной сети для соответствующего выбора в среде принятия решений. PESTLE - один из инструментов стратегического управления, предназначенный для анализа факторов внешней макросреды (External macro environment analysis), влияющих на предприятие. В этом анализе внешняя среда организации исследуется в шести различных измерениях: политика, экономика, социокультурная сфера, технологии, экологические и правовые факторы. Факторы PESTLE обычно выходят за рамки того, на что организация может напрямую влиять [13]. Соответственно, речь в данном исследовании идёт о решении актуальной задачи формализации и интеллектуального анализа слабоструктурированной информации о факторах, оказывающих существенное влияние на деятельность промышленной организации.

\section{Методология}

Для проведения исследования первоначально был использован процесс группового иерархического анализа. Процесс иерархического анализа - один из многоцелевых методов принятия решений [14]. Этот метод используется, когда действие по принятию решения сталкивается с несколькими конкурирующими вариантами и критериями принятия решения. Предлагаемые критерии могут быть количественными и качественными. В основе этого метода принятия решений лежит попарное сравнение, определяющее вес каждого фактора с точки зрения конкурирующих вариантов. Логика процесса иерархического анализа объединяет матрицы парных сравнений для принятия оптимального решения. Использование процесса иерархического анализа в групповых решениях позволяет не только сохранить преимущества методов группового принятия решений, но и устранить их недостатки (такие как скорость, стоимость и монотонность). И это поможет руководителю, принимающему решения, принять лучшее решение с учётом мнения всех участников.

Предлагаемый к реализации подход поддерживает принятие многокритериальных решений (Multi Criteria Decision Making) [15] в системах поддержки принятия решений с использованием методов оптимизации, математического планирования. В отличие от математических подходов, экспертные системы включают теории перцептивных решений в рабочую модель больше, чем математическую модель. При таком подходе проблема не обязательно должна быть смоделирована математически. Предпринимается попытка прийти к серии умных выводов в отсутствие полной информации и в отсутствие полной структуры проблемы. Экспертные системы в их продвинутой форме обладают способностью учиться и использовать прошлые ошибки, чтобы принимать более правильные решения на будущее. Экспертные системы основаны на хранении и выводе знаний, поэтому их также называют системами, основанными на знаниях [16]. 
Для определения степени и серьёзности воздействия факторов, индикаторов и критериев использовался процесс иерархического анализа, и, исходя из количества и серьёзности неопределённостей, в расчётах учитывалось принятие риска. В качестве объекта рассмотрения в данной работе выступал один из факторов инструмента стратегического управления PESTEL - политический фактор. В этой части основные использованные методы исследования - статистические и описательные. Затем с помощью метода нейронной сети существующие индикаторы были сгруппированы, что позволило оценить потенциал предложенного подхода в повышении качества принятия стратегических решений по развитию промышленных предприятий.

\section{Результаты}

В данном исследовании и анализе рассматривается только модуль «Политика» обозначенного инструмента стратегического управления, его цель состоит в том, чтобы предсказать, насколько успешно экономическая производственная система будет действовать с политической точки зрения на основе показателей эффективности за предыдущие годы. Для решения данной задачи требуется сформировать как можно более полный информационный массив по странам. В целом, за основу принимается предположение о том, что чем более благоприятным является политический фактор, тем более подходящим выступает окружающее пространство для деятельности промышленных предприятий. Для исследования использовались только общедоступные источники данных. Следующие описанные ниже источники данных содержали диапазон исторических данных с 2013 г. по 2017 г. для 108 стран (табл. 1).

Важно отметить, что перечисленные факторы могут меняться, оказывая влияние на функционирование промышленных предприятий, но при этом изменения носят в рассматриваемой сфере не радикальный характер в большинстве случаев, так как учитываются сводные данные, а не отдельные составляющие политического фактора.

Обработка данных - это важный шаг при разработке любого алгоритма машинного обучения [17], особенно для того, чтобы избежать действия правила «мусор на входе - мусор на выходе» (это понятие в информатике заключается в том, что ошибочные или бессмысленные входные данные производят бессмысленный вывод или «мусор») [18]. Если используются неверные или некачественные данные для изучения любой модели машинного обучения, это окажет прямое влияние на качество прогнозов.

Во-первых, как показано на рис. 1, для каждого источника было рассчитано среднее значение всех факторов, учитываемых этим источником данных за год. Например, для мировых показателей управления (WGI) - среднее значение таких индексов, как Участие общественности и подотчётность (Voice and Accountability); Политическая стабильность; Отсутствие насилия; Эффективность правительства; Качество регулирования и другие данные должны ежегодно фигурировать в базе данных WGI. 
Политические факторы в PESTEL анализе

\begin{tabular}{|c|c|c|}
\hline 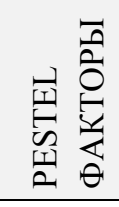 & 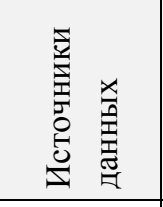 & Описание \\
\hline \multirow[b]{3}{*}{ 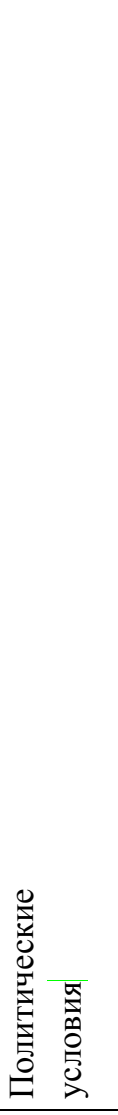 } & 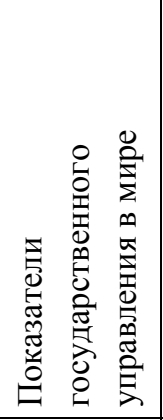 & $\begin{array}{l}\text { В проекте Worldwide Governance Indicators (WGI) представлены } \\
\text { агрегированные и индивидуальные показатели управления для более } \\
\text { чем } 200 \text { стран и территорий по шести измерениям управления: } \\
\text {-Участие общественности и подотётность } \\
\text {-Политическая стабильность и отсутствие насилия } \\
\text {-Эффективность правительства } \\
\text {-Нормативное качество } \\
\text {-Верховенство закона } \\
\text {-Контроль коррупции }\end{array}$ \\
\hline & 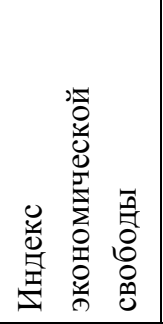 & $\begin{array}{l}\text { Wall Street Journal и The Heritage Foundation измеряют десять } \\
\text { переменных и оценивают более } 180 \text { стран. Индекс оценивает } \\
\text { государственную политику и экономические условия в } 186 \text { странах. } \\
\text { С момента его создания в } 1995 \text { г. Индекс, ежегодный межстрановой } \\
\text { анализ, проводимый The Heritage Foundation, отслеживает прогресс } \\
\text { экономической свободы во всём мире и измеряет реальное влияние } \\
\text { продвижения экономической свободы. }\end{array}$ \\
\hline & 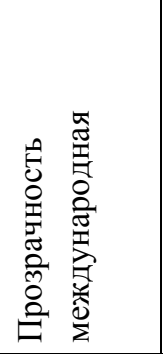 & $\begin{array}{l}\text { Международная Прозрачность (Transparency International) ежегодно } \\
\text { публикует «Глобальный отчёт о коррупции», «Индекс плательщиков } \\
\text { взяток» и «Индекс восприятия коррупции», в которых страны } \\
\text { ранжируются по степени распространённости коррупции среди } \\
\text { государственных служащих. }\end{array}$ \\
\hline 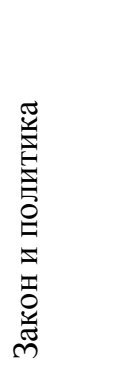 & 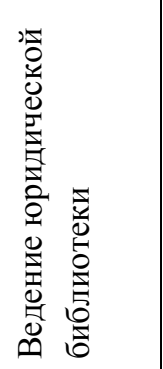 & $\begin{array}{l}\text { Онлайн-сборник законов и постановлений о промышленных } \\
\text { редприятиях. Ссылки на официальные правительственные источники } \\
\text { законов о труде, налогах, торговле, собственности, банковском деле, } \\
\text { предприятиях, гражданском процессе и банкротстве. Часть проекта } \\
\text { «Doing Business» Всемирного банка. }\end{array}$ \\
\hline
\end{tabular}

Источник: составлено автором 
Сохейли Могадам Сейед Махди. Совершенствование аналитического инструмента PESTEL в системе стратегического управления промышленным предприятием

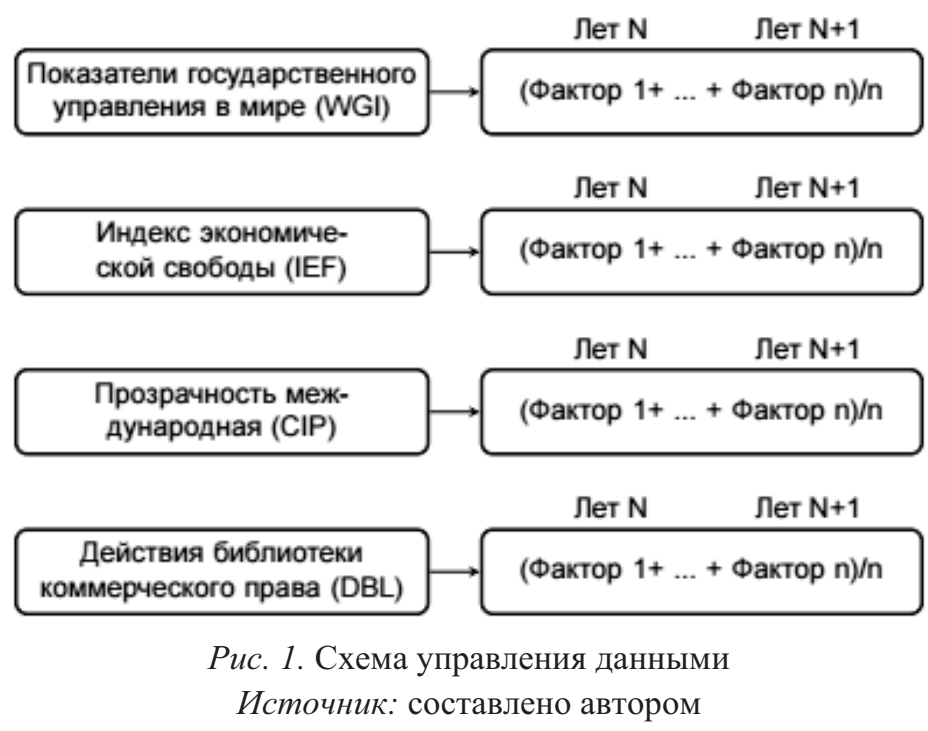

Во-вторых, как показано на рис. 2, среднее значение индексов, полученных из четырёх различных баз данных за соответствующий год, использовалось в качестве входных данных, а среднее значение индексов, полученных в следующем году, использовалось в качестве целевого показателя, являющегося результатом, который следует прогнозировать.

\begin{tabular}{|c|c|c|c|c|c|c|c|}
\hline \multicolumn{4}{|c|}{ Вход } & \multicolumn{4}{|c|}{ Выход } \\
\hline WGI & IEF & CIP & DBL & WGI & IEF & CIP & DBL \\
\hline Лет $\mathrm{N}$ & Лет $\mathrm{N}+1$ & Лет $\mathrm{N}$ & (Лет $\mathrm{N}+1$ & Лет $\mathrm{N}$ & Лет $\mathrm{N}+1$ & Лет $\mathrm{N}$ & Лет $\mathrm{N}+1$ \\
\hline
\end{tabular}

Рис. 2. Входная и целевая схемы

Источник: составлено автором

Для построения нейронной сети использовалась библиотека Python для глубокой нейронной сети KERAS. Эта открытая нейросетевая библиотека предоставляет различные модули для создания и изучения нейронных сетей. Такие модули, как нейронные уровни, функции стоимости, оптимизаторы, схемы инициализации, функции активации и другие можно комбинировать для создания новых моделей. Следовательно, модель нейронной сети может быть построена в соответствии с шагами, обозначенными на рис. 3 .

Для обучения модели данные необходимо разделить на две разные категории: предикторы X и цели Y. В разделе DML были произведены все манипуляции для достижения нужной структуры (рис. 4). 


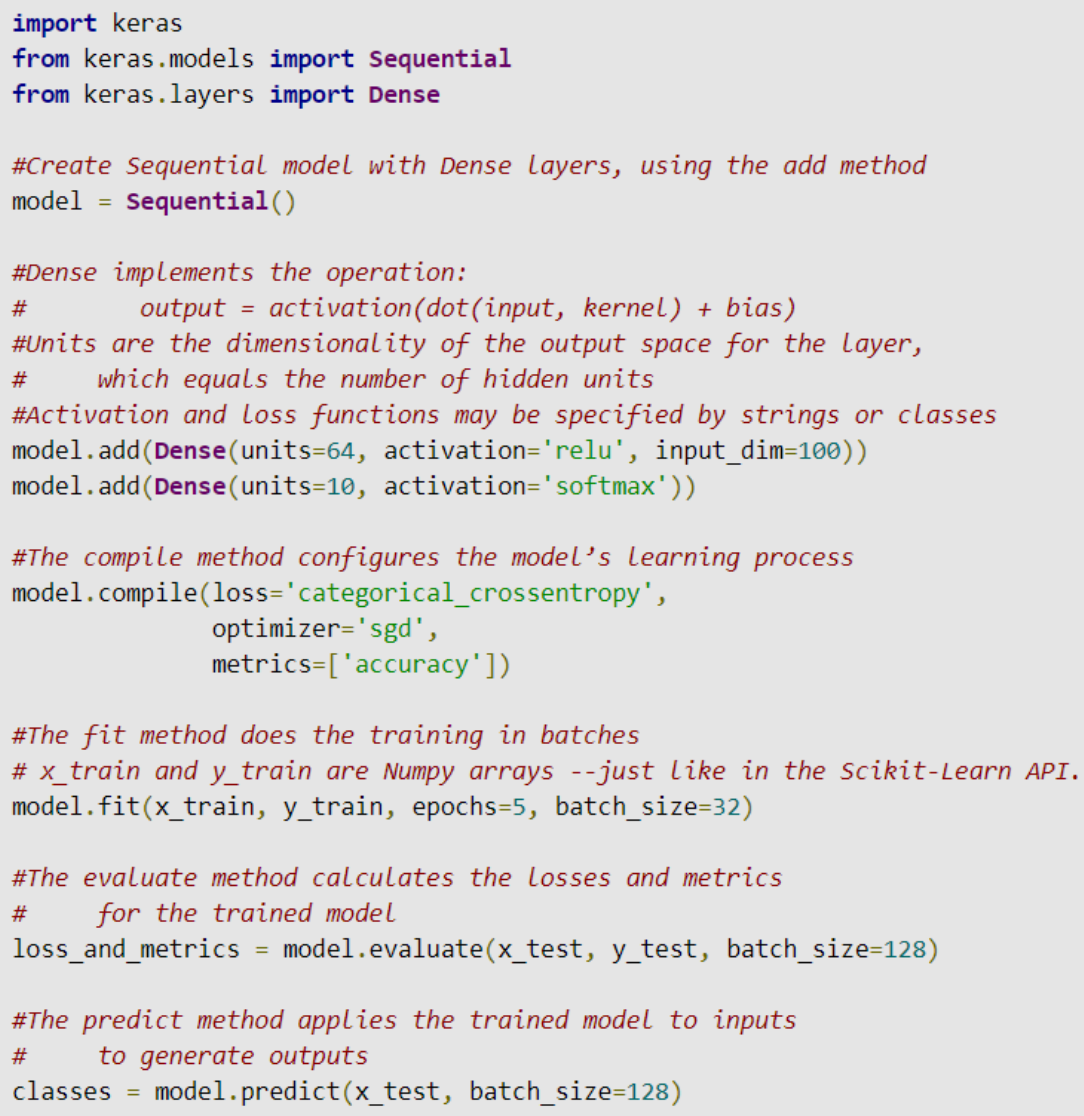

Puc. 3. Построение нейронной сети с помощью KERAS

Источник: составлено автором на основе материалов исследования

\begin{tabular}{|c|c|c|c|c|c|c|}
\hline \multirow{2}{*}{$\begin{array}{l}\text { In }[50]: \\
\text { Out }[50]:\end{array}$} & \multicolumn{6}{|c|}{ political.head() } \\
\hline & & wgi_x & index_x & cpi_x & dbll_x & $\mathbf{Y}$ \\
\hline & 0 & 45.038530 & 65.2 & 31 & 90.19 & 60.043712 \\
\hline & 1 & 25.663619 & 49.6 & 36 & 73.74 & 45.703716 \\
\hline & 2 & 15.442524 & 47.3 & 23 & 53.82 & 34.471750 \\
\hline & 3 & 39.831935 & 46.7 & 34 & 72.58 & 46.611185 \\
\hline & 4 & 46.092087 & 69.4 & 36 & 93.39 & 60.450627 \\
\hline
\end{tabular}

Рис. 4. Окончательная структура данных

Источник: составлено автором на основе результатов исследования 
Сохейли Могадам Сейед Махди. Совершенствование аналитического инструмента PESTEL в системе стратегического управления промышленным предприятием

Как было отмечено ранее, индексы года (YEAR N) использовались в качестве предикторов, а индексы года после (YEAR N + 1) использовались в качестве цели (табл. 2).

Таблица 2

Годы прогнозирования и целей

\begin{tabular}{|l|l|}
\hline \multicolumn{1}{|c|}{ Предикторы (Х) } & \multicolumn{1}{c|}{ Цель (Y) } \\
\hline 2013 & 2014 \\
\hline 2014 & 2015 \\
\hline 2015 & 2016 \\
\hline 2016 & 2017 \\
\hline
\end{tabular}

Источник: составлено автором

Пример на рис. 3 взят из задачи классификации, и эта разработка носит описательный характер. Следовательно, при компиляции функции потерь, которая будет использоваться, будет среднеквадратичная ошибка, и оптимизатор весов будет выбран путём оценки производительности SGD, стохастического градиентного спуска, в сравнении с оценкой адаптивного момента Адама, которая является расширением стохастического градиентного спуска.

Сравнение производительности проводилось в ходе исследования для проверки того, какой оптимизатор является лучшим для решения поставленной задачи. В связи с этим были использованы две модели: модель 1 была обучена с использованием оптимизатора ADAM; модель 2 - использующая оптимизатор SGD. Несоответствие диапазона валидации между моделью 1 (красный) и моделью 2 (синий) (рис. 5) даёт неправильное представление о том, что вторая модель начинается с потерь, близких к нулю, когда действительно он начинается примерно с 13 и заканчивается потерей валидации: 1.9023 .

Использование модели 2 для прогнозирования показателей показало, что эта модель имеет самый низкий балл проверки, именно он используется для прогнозирования индексов 2018 г. с использованием индексов 2017 г. в качестве предикторов (рис. 6).

Учитывая величину потерь, полученную в модели, использованной для прогнозирования, которая составляла 1,9023, полученные прогнозы далеки от достоверности. Согласно прогнозам, полученным с помощью модели 2, в первую десятку входят страны, перечисленные в таблице ниже. Если сравнивать с данными за 2017 год, динамика топ-10 стран практически такая же (табл. 3).

Чтобы сделать визуализацию данных более простой и быстрой для восприятия, в PowerBI было импортировано прогнозирование политической ситуации. Визуализация отфильтрована по карте для 10 стран с лучшими индексами, в результате чего получено изображение ниже (рис. 7). 


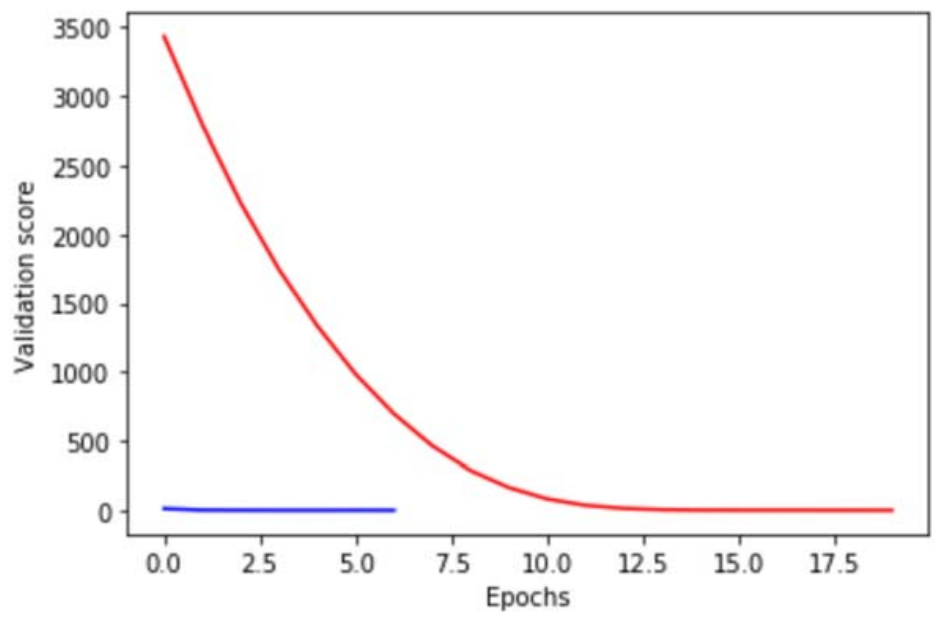

Puc. 5. График сравнения оценок валидации модели 1 и модели 2 Источник: составлено автором

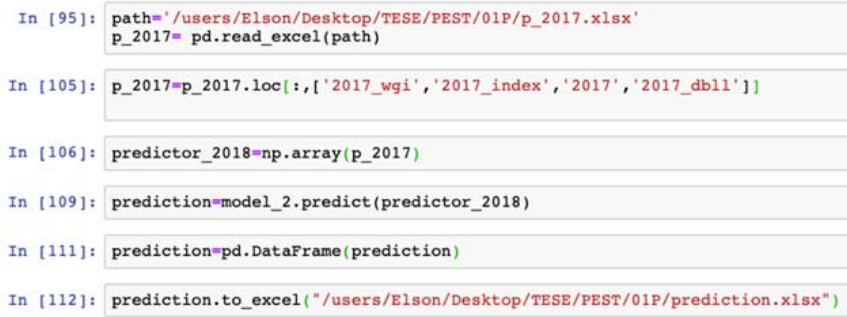

Puc. 6. Реализации прогноза политической ситуации в python Источник: составлено автором

\section{Сравнение реальных значений политической ситуации 2017 г.}

\section{с прогнозами на 2018 г.}

\begin{tabular}{|l|l|l|}
\hline \multicolumn{1}{|c|}{ Страна } & $2017-$ Реальный & \multicolumn{1}{c|}{2018 - Прогнозный } \\
\hline Новая Зеландия & 92.75463086 & 91.63370514 \\
\hline Сингапур & 89.50503246 & 88.39645386 \\
\hline Канада & 88.46305147 & 87.66313171 \\
\hline Швейцария & 87.99027132 & 87.02622986 \\
\hline Норвегия & 87.66101293 & 86.59942627 \\
\hline Швеция & 87.1332298 & 86.07897949 \\
\hline Финляндия & 86.96527822 & 85.85818481 \\
\hline Нидерланды & 86.60231473 & 85.72468567 \\
\hline Австралия & 86.48155923 & 85.94795227 \\
\hline
\end{tabular}

Источник: составлено автором 


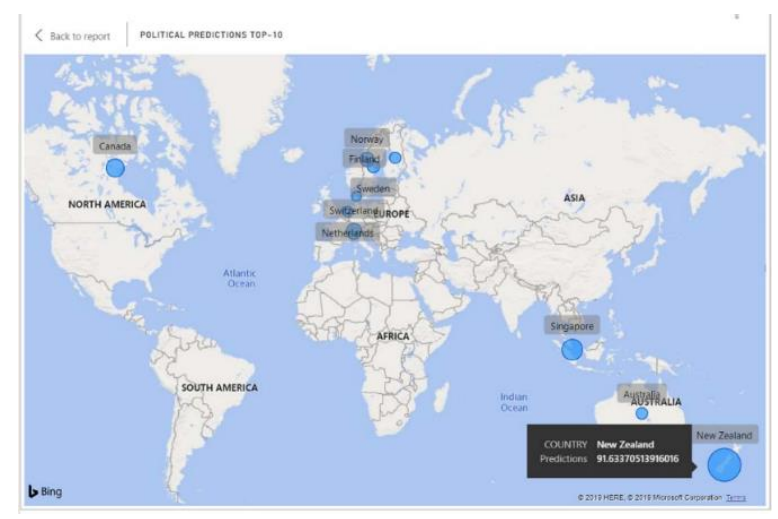

Puc. 7. Карта Power BI с топ-10 прогнозов политической ситуации Источник: составлено автором

\section{Заключение}

Таким образам, эффективность нейронной сети прямо пропорциональна набору данных, который использовался для её построения. Принимая во внимание диапазон лет, используемых для обучения этой модели, с 2013 г. по 2016 г., и высокие значения потерь при проверке, полученные с помощью этой модели, прогнозы далеки от правильности. Однако, учитывая полученные результаты, любое предприятие, имеющее средства для приобретения подходящих наборов данных по экономическим, политическим, социальным, правовым и экологическим вопросам из надежных источников и инвестируя в построение модели с более сложной социальной математикой для машинного обучения, несомненно, выиграет от наличия такого актива в своих информационных системах.

Прямой поток входных данных, определённых аналитиками, может дать точную картину политической ситуации в каждой стране во всём мире в режиме реального времени, что позволит лицам, принимающим решения, предпринять любые необходимые действия. К числу таких действий может относиться корректировка содержания стратегий на промышленном предприятии в случае каких-либо внезапных изменений в любой из стран. Благодаря новым знаниям промышленные предприятия могут получить конкурентное преимущество, укрепить свои стратегические позиции и обеспечить адаптивность и качество системы стратегического управления.

\section{Список литературы}

1. Gunn, R. Strategic tools: an empirical investigation into strategy in practice in the UK / R. Gunn, W. Williams // Strategic Change. - 2007. - Vol. 16, no. 5. - P. 201-216.

2. Cambridge Handbook of strategy as practice / D. Golsorkhi, L. Rouleau, D. Seidl, E. Vaara. - Cambridge : Cambridge University Press, 2010. - 20 p. 
3. Afonina, A. Investigation of strategic management tools and techniques / A. Afonina, V. Chalupský // Acta Universitatis Agriculturae et Silviculturae Mendelianae Brunensis. - 2013. - Vol. 61, no. 4. - P. 833-840.

4. Reif, W. E. The Manager's guide to strategic planning tools and techniques / W. 0E. Reif, J. L. Webster, J. S. Bracker // Planning Review. - 1989. - Vol. 17, no. 6. P. $4-48$.

5. Pasanen, M. Strategic management tools and techniques in SMEs // SSRN Electronic Journal. - 2011. - P. 1-8. - URL: https://papers.ssrn.com/sol3/papers.cf m?abstract_id=1867897 (дата обращения: 30.05.2021).

6. Frost, F. A. The use of strategic tools by small and medium-sized enterprises: an Australasian study / F. A. Frost // Strategic Change. - 2003. - Vol. 12, no. 1. - P. 49-62.

7. Yachmeneva, V. Factors influencing the enterprise innovation // Econtechmod an International Quarterly Journal. - 2014. - Vol. 1, no. 1. - P. 133-138.

8. The impact of political factors' perception on suitability of international business environment: The case of startups / L. Kozubikova, A. Kotaskova, J. Dvorsky, A. Kljucnikov // Economics \& Sociology. - 2019. - Vol. 12, no. 1. - P. 61-79.

9. Tonelli, M. Unstructured strategic decision-making processes: CRE decisionmaking in the Italian consulting industry : thesis dis. ... $\mathrm{PhD} / \mathrm{M}$. Tonelli. - Queensland, 2009. - 370 p. URL: file://C:/Users/youhi/Downloads/Marcello_Tonelli_Thesis.pdf (дата обращения: 30.05.2021).

10. Moore, F. A comparison of information systems - EISs, DSS, and MISs. 2017. - URL: file://C:/Users/youhi/Downloads/MooreFMIS7003-7_FinalPDF_Fahme enaOdettaMoore.pdf (дата обращения: 30.05.2021).

11. Eom, S. A survey of decision support system applications (1995-2001) / S. Eom, E. A. Kim // Journal of the Operational Research Society. - 2006. - Vol. 57. - P. 1264-1278.

12. Beemer, B. A. Advisory systems to support decision making / B. A. Beemer, D. G. Gregg // Handbook on Decision Support Systems 1. - Springer, 2008. - P. 511-527.

13. Thompson J. L., Scott J. M., Martin F. Strategic management: awareness and change - 2019. - 9th edition. - Published 13 December 2019. - URL: https://www.rese archgate.net/publication/337465389_Strategic_Management_Awareness_and_Change_9t h_Edition_-_Published_13_December_2019.

14. Khan, A. Analytical hierarchy process (ahp) and analytic network process methods and their applications: a twenty year review from 2000-2019 / A. Khan, Y. Ali // International Journal of the Analytic Hierarchy Process. - 2020. - Vol. 12, iss. 3. - P. 2020-1936.

15. Yüksel, İ. Developing a multi-criteria decision making model for PESTEL analysis // International Journal of Business and Management. - 2012. - Vol. 7, iss. 24. P. 52-66.

16. Power, D. J. Decision support systems: concepts and resources for managers / D. J. Power. - [S. 1.] : Greenwood Publishing Group, 2002. - 284 p. 
17. Li, S. Research on data mining technology based on machine learning algorithm // Journal of Physics: Conference Series. - 2019. - Vol. 1168. - Art. no. 032132.

18. Artificial Intelligence in Automated Sorting in Trash Recycling / B. S. Costa, A. C. S. Bernardes, J. V. A. Pereira, V. H. Zampa [et al.]. - DOI 0.5753/eniac.2018.4416// XV Encontro Nacional de Inteligência Artificial e Computacional (ENIAC). - São Paulo, 2018. - URL: file:///C:/Users/youhi/D ownloads/sbctemplate-v2\%20(1).pdf (дата обращения: 30.05.2021).

\section{References}

1. Gunn R., Williams W. Strategic tools: an empirical investigation into strategy in practice in the UK / R. Gunn, W. Williams. Strategic Change, 2007, vol. 16, no. 5, pp. 201-216.

2. Golsorkhi D., Rouleau L., Seidl D., Vaara E. Cambridge Handbook of Strategy as Practice. Cambridge: Cambridge University Press, 2010. 20 p.

3. Afonina A., Chalupský V. Investigation of strategic management tools and techniques. Acta Universitatis Agriculturae et Silviculturae Mendelianae Brunensis, 2013, vol. 61 , no. 4 , pp. $833-840$.

4. Reif W. E., Webster J. L., Bracker J. S. The Manager's guide to strategic planning tools and techniques. Planning Review, 1989, vol. 17, no. 6, pp. 4-48.

5. Pasanen M. Strategic Management Tools and Techniques in Smes. SSRN Electronic Journal, 2011, pp. 1-8. Available at: ttps://papers.ssrn.com/sol3/papers.cf m?abstract_id $=1867897$ (accessed 30 May 2021).

6. Frost F. A. The use of strategic tools by small and medium-sized enterprises: an Australasian study. Strategic Change, 2003, vol. 12, no. 1, pp. 49-62.

7. Yachmeneva V. Factors influencing the enterprise innovation. Econtechmod. an International Quarterly Journal, 2014, vol. 1, no. 1, pp. 133-138.

8. Kozubikova L., Kotaskova A., Dvorsky J., Kljucnikov A. The impact of political factors' perception on suitability of international business environment: The case of startups. Economics \& Sociology, 2019, vol. 12, no. 1, pp. 61-79.

9. Tonelli M. Unstructured strategic decision-making processes : CRE decision-making in the Italian consulting industry. Thesis dis. PhD. Queensland, 2009. 370 p. Available at: file://C:/Users/youhi/Downloads/Marcello_Tonelli_Thesis.pdf (accessed 30 May 2021).

10. Moore F. A comparison of information systems - EISs, DSS, and MISs. 2017. Available at: file:///C:/Users/youhi/Downloads/MooreFMIS7003-7_FinalPDF_Fahmee naOdettaMoore.pdf (accessed 30 May 2021).

11. Eom S., Kim E. A Survey of decision support system applications (1995-2001). Journal of the Operational Research Society, 2006, vol. 57, pp. 1264-1278.

12. Beemer B.A., Gregg D.G. Advisory systems to support decision making. Handbook on Decision Support Systems 1. Springer, 2008, pp. 511-527. 
13. Thompson J. L., Scott J. M., Martin F. Strategic management: awareness and change. Strategic management. - 2019.

14. Khan A., Ali Y. Analytical hierarchy process (AHP) and analytic network process methods and their applications: a twenty year review from 2000-2019. International Journal of the Analytic Hierarchy Process, 2020, vol. 12, iss. 3, pp. 2020-1936.

15. Yüksel İ. Developing a multi-criteria decision making model for PESTEL analysis. International Journal of Business and Management, 2012, vol. 7, iss. 24, pp. 52-66.

16. Power D. J. Decision Support Systems: Concepts and Resources for Managers. Greenwood Publishing Group, 2002. 284 p.

17. Li S. Research on data mining technology based on machine learning algorithm. Journal of Physics: Conference Series, 2019, vol. 1168, art. no. 032132.

18. Costa B. S., Bernardes A. C. S., Pereira J. V. A., Zampa V. H. et al. Artificial Intelligence in Automated Sorting in Trash Recycling. XV Encontro Nacional de Inteligência Artificial e Computacional (ENIAC). São Paulo, 2018. Avaiable at: file:///C:/Users/youhi/Downloads/sbc-template-v2\%20(1).pdf (дата обращения: 30 Мау 2021). DOI 0.5753/eniac.2018.4416. 